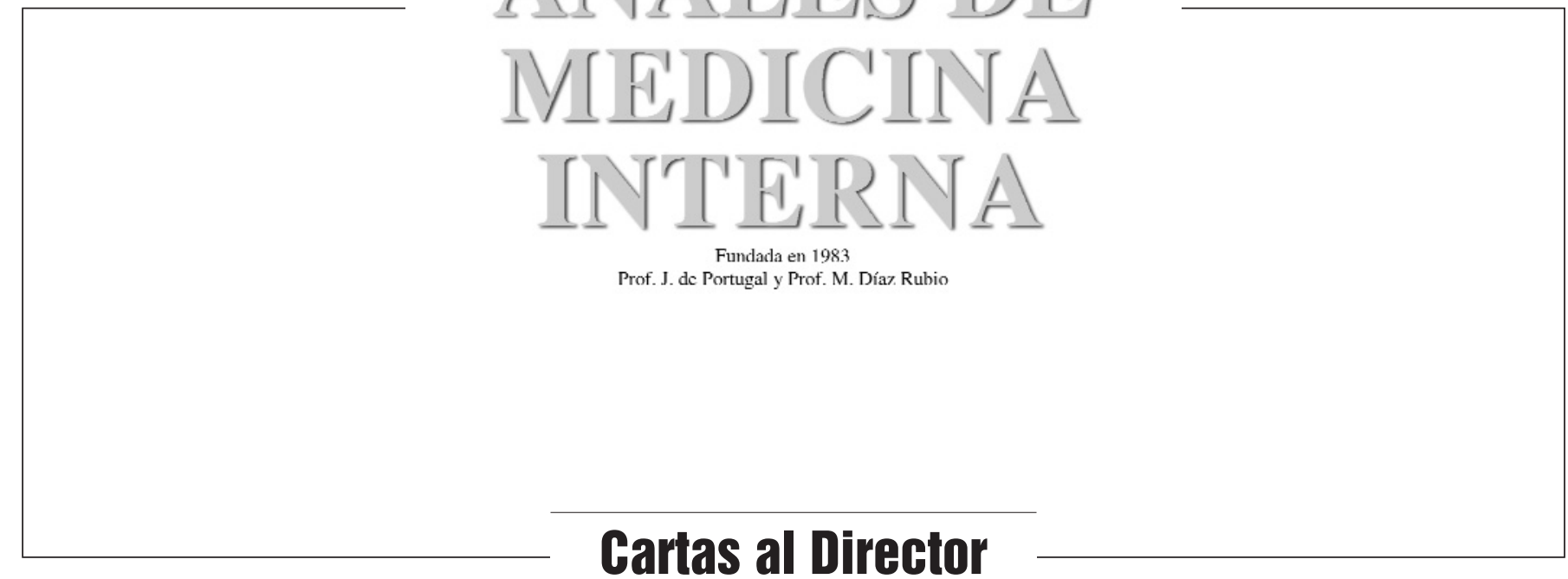

\section{Endocarditis subaguda asintomática en paciente en hemodiálisis}

\section{Sr. Director:}

Están sobradamente descritos los múltiples factores por los que los pacientes renales sometidos a hemodiálisis tienen predisposición a sufrir endocarditis. Entre estos, destacan el estado de inmunosupresión que padecen, las punciones repetidas de la fístula arteriovenosa, catéteres temporales o permanentes para la diálisis, valvulopatías de base, calcificaciones valvulares relacionadas con el hiperparatiroidismo que constituyen un buen nido para la infección, etc. (1-3).

Por otro lado, el diagnóstico de una endocarditis en este tipo de pacientes está dificultado por una serie de circunstancias, como su alta incidencia de bacteriemias, comorbilidad elevada etc.

Para el diagnóstico de una endocarditis en este tipo de pacientes, los parámetros utilizados no son distintos que para el resto de la población, siendo los criterios de Duke los que generan mayor consenso $(4,5)$.

Presentamos un caso de un paciente, varón de 67 años, con una insuficiencia renal crónica secundaria a amiloidosis primaria (sín afectación cardiaca en estudios ecocardiográficos realizados previamente), que había iniciado hemodiálisis dos meses antés por catéter yugular derecho que había sido retirado cuatro semanas atrás por cuadro febril y por presentar fístula arteriovenosa funcionante, al que, permaneciendo asintomático, se le realiza ecocardiografía rutinaria de cara a su inclusión en lista de espera para trasplante renal, y en el que se objetiva prolapso de velo posterior mitral junto con imagen vibrátil sobreañadida de gran tamaño que genera regurgitación mitral severa, y que es contrastada con ecocardiografía tranesofágica.

A la vista del hallazgo y a pesar de permanecer asintomático, se decide ingreso hospitalario así como realización de estudios para determinar el posible origen del cuadro. Entre las pruebas realizadas, se aislaron staphylococcus epidermidis en hemocultivos seriados, así como elevación importante de los reactantes de fase aguda, leucocitosis con desviación izquierda y esplenomegalia.

El paciente fue tratado durante seís semanas con vancomicina administrada durante las sesiones de hemodiálisis, permanecien- do en todo momento asintomático.

Transcurrido este tiempo, la imagen ecocardiográfica había disminuido de tamaño, y las alteraciones analíticas y la esplenomegalia habían desaparecido. Se desestimó su derivación a Cirugía Cardiaca.

Nos parece interesante el caso, por insistir en la alta incidencia de endocarditis en pacientes en hemodiálisis (se estima que hasta un 5\% de estos pacientes pueden padecerla) (1) sus factores favorecedores y dificultad diagnóstica (6-8), y sobre todo como un paciente con un cuadro grave ha podido permanecer sín clínica alguna durante varias semanas realizando el diagnóstico de forma fortuita. Probablemente el origen del cuadro hubiese estado en el catéter yugular que había tenido, que fue retirado por fiebre y tratado entonces con una dosis única de vancomicina, sín chequeo bacteriológico, y con mejoría clínica desde aquel mismo momento.

\section{R. Álvarez Navascués, C. J. Hsieh Ching ${ }^{1}$, I. Möller ${ }^{2}$, L. Qui- ñones Ortiz, J. Guerediaga Madariaga}

Servicios de Nefrología, 'Medicina Familiar y Comunitaria y ${ }^{2}$ Cardiología. Hospital San Agustín. Avilés

1. Maraj S, Jacobs LE, Maraj R, Kotler MN.Bacteriemia and infective endocarditis in patients on hemodialysis. Am J Med Sci 2004; 327: 242 249.

2. Hoen B. Infective endocarditis: a frequent disease in dialysis patients. Nephrol Dial Transplant 2004; 19: 1360-1362.

3. Perkovic V, Hunt D, Griffin SV, Du Plessis M, Becker GJ. Accelerated progression of calcific aortic stenosis in dialysis patients. Nephron Clin Pract 2003; 94:40-45.

4. Li, JS, Sexton, DJ, Mick, $\mathrm{N}$ et al. Proposed modifications to the duke criteria for the diagnosis of infective endocarditis. Clin Infect Dis 2000; 30: 633-637.

5. Bayer AS, Ward JL, Ginzton LE, Shapiro SM. Evaluation of new clinical criteria for the diagnosis of infective endocarditis. Am J Med 1994; 96: 211-215.

6. Deshpande KS, Hatem C, Ulrich HL, Currie BP, Aldrich TK, BryanBrown CW, Kvetan V. The incidence of infectious complications of central venous catheters at subclavian, internal jugular, and femoral sites in an intensive care unit population. Crit Care Med 2005; 33:13-20.

7. Whitener C, Caputo GM, Weitekamp MR, Karchmer AW. Endocarditis due to coagulase-negative staphylococci: Microbiologic, epidemiologic and clinical considerations. Infect Dis Clin North Am 1993; 7: 81-89. 
8. Rupp ME. Coagulase-negative staphylococcal infections: an update regarding recognition and manegement. Curr Clin Top Infect Dis 1997; 17: $51-54$.

\section{Pericarditis purulenta por Streptococcus agalactiae}

\section{Sr. Director:}

La pericarditis purulenta se ha constituido en una entidad muy infrecuente desde el inicio de la era antibiótica. Para su definición es requisito indispensable el hallázgo de líquido macro o microscopicamente purulento en el saco pericárdico, por lo que la vía diagnóstica pasa irremediablemente por la pericardiocentesis (1).

Los mecanismos para su desarrollo son variados : extensión directa desde foco infeccioso intratorácico, foco miocárdico, infección directa por trauma o cirugía torácica, extensión desde foco infeccioso subdiafragmático o por diseminación hematógena (2).

El Streptococcus agalactiae o Streptococcus grupo B (SGB), clasicamente se ha asociado con enfermedades graves en mujeres embarazadas y recién nacidos.

Las infecciones invasoras por SGB en adultos no obstétricos han sufrido un significativo aumento en los últimos años, alcanzando incidencias de 4-7 casos por 100.000 adultos/año $(3,4)$.

Se describen una serie de factores de riesgo asociados, como pueden ser la hepatopatía, diabetes mellitus, alcoholismo, neoplasias, cardiopatías de base, insuficiencia renal, alteraciones del SNC, VIH y otras, destacando entre todas ellas las cuatro primeras $(4,5)$.

Presentamos aquí el caso de un paciente, varón de 47 años, que acude a nuestro hospital refiriendo malestar general de unos 8 días de evolución, con fiebre oscilante de hasta $40^{\circ} \mathrm{C}$, con fases de 48 horas en las que permanecía afebril.

No manifestaba clínica por aparatos, salvo una pequeña lesión eritematopustulosa en región pretibial izquierda que achacaba a traumatismo casero.

Entre sus antecedentes personales, destacaba enolismo importante así como intolerancia a los hidratos de carbono para la que no observaba cuidado alguno.

En la exploración física a su ingreso (y durante todo él) permanecía afebril, consciente y orientado en todo momento, ligero tinte ictérico, con presión arterial de 100/50 (no se la controlaba antes de su ingreso), auscultación cardiopulmonar normal, abdomen con hepatoesplenomegalia sín evidencia de líquido libre, y destacaba una lesión en región pretibial izquierda eritemato-pustulosa no supurativa.

En los estudios realizados, leucocitosis marcada (22.000 leucocitos) con desviación izquierda, plaquetopenia $\left(50.000 / \mathrm{mm}^{3}\right)$ y tasa de protrombina del $45 \%$. Por otro lado, alteración importante de la bilirrubina y resto de pruebas de función hepática (bilirrubina 5,6 mg/dl, TGP $570 \mathrm{UI} / \mathrm{L}$, TGO $769 \mathrm{UI} / \mathrm{L}$, FA $408 \mathrm{UI} / \mathrm{L}$ ), así como datos de insuficiencia renal (urea 200, Cr 4,5 mg/dl). En la radiografía de tórax cardiomegalia sín signos de redistribución ni condensaciones. La ecografía abdominal resultó inespecífica (hepatoesplenomegalia).

El cuadro se interpretó como posible hepatopatía crónica de origen etílico, así como cardiopatía dilatada, y posible descompensación en relación con sepsis de origen no claro, con fracaso renal agudo asociado.

Se demoró el chequeo bacteriológico y el inicio de tratamiento antibiótico en espera de pico febril que no se produjo.

En las 24 horas siguientes a su ingreso, se observó inestabilidad hemodinámica, por lo que se realizó ecocardiografía transtorácica en la que se descartaba endocarditis, objetivando derrame pericárdico que en aquel momento no comprometía la funcionalidad cardiaca.

Asistimos a un deterioro general progresivo, por lo que se decide su ingreso en UCI, donde se realiza hemodiálisis de soporte así como pericardiocentesis, en la que se obtienen $800 \mathrm{cc}$ de líquido purulento.

Ya se había iniciado tratamiento antibiótico con amoxicilinaclavulánico y realizado chequeo bacteriológico.

En las horas siguientes, desarrolló fracaso multiorgánico siendo exitus.

Posteriormente se aisló Streptococcus agalactiae en el drenaje pericárdico así como en el aspirado de su lesión pretibial izquierda.

Los hemocultivos resultaron negativos (ya se había iniciado pauta antibiótica), así como el resto de los estudios realizados.

Parece tratarse de una sepsis por SGB, con foco de entrada en lesión cutánea y fracaso multiorgánico con piopericarditis por diseminación hematógena.

Existen cuatro casos descritos en la literatura de piopericarditis por SGB. En tres de ellos se hace referencia a la extensión directa desde foco miocárdico (abscesos miocárdicos y endocarditis) (6). No hemos encontrado casos que hagan referencia a diseminación hematógena por este gérmen al saco pericárdico.

Aunque se trata de un gérmen en general multisensible, los casos de infección diseminada por SGB presentan una alta tasa de mortalidad. Nuestro paciente presentaba varios de los factores de riesgo asociados a su alta virulencia (alcohol, probable hepatopatía de base, diabetes,...).

Por otro lado, el hallázgo de una pericarditis purulenta representa una rara entidad hoy en día, con una muy elevada mortalidad. Todavía en la actualidad, la mayor parte de estos diagnósticos se realizan postmorten (7).

Por lo tanto, se trata de un caso raro en cuanto a la presentación, etiología y fisiopatología, de difícil sospecha clínica, y mal pronóstico cuando el diagnóstico se realiza una vez instaurado un fracaso multiorgánico, a pesar de tratarse de un germen, en principio poco peligroso.

\section{R. Álvarez Navascués, M. Sánchez Cembellín', I. Carrio Montes ${ }^{1}$, L. Quiñones Ortiz, J. Guerediaga Madariaga}

Sección de Nefrología y ${ }^{1}$ Servicio de Medicina Interna. Hospital San Agustín. Avilés

1. Keersmaekers T, Elshot SR, Sergeant PT. Primary bacterial pericarditis. Acta Cardiol 2002; 57: 387-389.

2. Sagrista-Sauleda J, Barrabes JA, Permanyer-Miralda G, Soler-Soler J. Purulent pericarditis:review of a 20-year experience in a general Hospital. J Am Coll Cardiol 1993; 15; 22: 1661-1665

3. Braunstein H, Tucker E, Gibson BC. Infections caused by unusual beta hemolytic strptococci. Am J Clin Pathol 1971; 55: 424-430.

4. Peirotti MG, Gonzalez Se, Littvik AM, Vacaflor L, Kassar MA, Moreno S, Bottiglieri MT. Group B streptococcal infections in adults, excluding genital infections. Rev Argent Microbiol 2002; 34: 226-229.

5. Genta PR, Dias ML, Janiszewski TA, Carvalho JP, Arai MH, Meireles LP. Streptococcus agalactiae endocarditis and giant Pyomyoma simulating ovarian cancer.South Med J 2001; 94: 508-511.

6. Abad C. Purulent abscess myocarditis with fatal outcome, caused by Streptococcus agalactiae. Med Clin (Barc) 1996; 106: 119.

7. Klacsmann PG, Bulkley BH, Hutchins GM. The changed spectrum of purulent pericarditis: An 86 year autopsy experience in 200 patients. Am J Med 1977; 63: 666-673. 


\section{Ginkgo biloba y hemorragia cerebral}

Sr. Director:

El Ginkgo biloba (GB) es un árbol originario del extremo Oriente, se considera un fósil viviente que no ha cambiado desde la época de los dinosaurios. Ha sido utilizado desde tiempo inmemorial en la medicina tradicional China. Fue traído a Europa y América en el siglo XVIII e introducido en la medicina occidental desde los años 70 (1).

En Occidente se viene usando desde hace años con distintas indicaciones: tinnitus, asma, enfermedades alérgicas, enfermedad arterial periférica, enfermedad de Raynaud, degeneración macular, glaucoma, como antioxidante para retrasar el envejecimiento y la más extendida parar preservar y mejorar la memoria (2).

En nuestro país está comercializado como solución oral con un extracto de Ginkgo biloba (EGb 761) y como cápsulas conteniendo polvo micronizado de hojas de GB. No está descrito en la ficha comercial efecto secundario alguno ni interacciones con otros fármacos, siendo por tanto un fármaco aparentemente inocuo.

Sin embargo en la literatura científica aparecen desde el año 199610 comunicaciones de complicaciones hemorrágicas, fundamentalmente intracraneales, asociadas a la toma de Ginkgo biloba (3).

A continuación describimos someramente dos casos de hemorragia cerebral en pacientes que tomaban ácido acetilsalicílico y extracto de Ginkgo biloba.

Varón de 73 años con antecedente de dislipemia, ex fumador de 40 paquetes/año diagnosticado hace 2 años de enfermedad de Alzheimer con TAC craneal entonces con atrofia córtico-subcortical y leucoaraiosis. Actualmente en tratamiento con extracto de Ginkgo biloba $80 \mathrm{mg} /$ día desde hace 2 años, ácido acetil salicílico (AAS) $100 \mathrm{mg} /$ día desde hace 2 años, vitamina E $200 \mathrm{mg} /$ día, galantamina $12 \mathrm{mg} / 12$ horas y pravastatina $10 \mathrm{mg} /$ día. De forma súbita sufre una hemiparesia izquierda objetivándose en el TAC craneal una hemorragia cerebral frontotemporal derecha; recuperándose en parte y quedándole como secuela mayor deterioro cognitivo (perdió 8 puntos del Mini Mental State Examination de Folstein) y paresia facial izquierda, retirándosele al alta el AAS pero no el extracto de GB.

Mujer de 83 años con antecedente de HTA, cardiopatía isquémica, diagnosticada de enfermedad de Alzheimer hace 2 años con TAC craneal entonces sin hallazgos. Actualmente en tratamiento con extracto de GB $80 \mathrm{mg} /$ día desde hace 2 años, AAS $100 \mathrm{mg} /$ día desde hace 4 años, donepezilo $5 \mathrm{mg} /$ día, diltiazem $240 \mathrm{mg}$ /día, loracepam $1 \mathrm{mg} /$ día, parche de nitroglicerina de $25 \mathrm{mg} /$ día, citalopram $20 \mathrm{mg} /$ día, pantoprazol $20 \mathrm{mg} /$ día, furosemida 77,6 mg/día y triamterene $25 \mathrm{mg}$ /día. Sufre de forma brusca un episodio de mareo con caída al suelo objetivándose hemorragia masiva bilateral cerebral abierta a ventrículos; evoluciona tórpidamente falleciendo en las 24 horas siguientes.

El extracto de Ginkgo biloba es un fármaco de amplio uso en nuestra población, muchas veces desconocido por los facultativos ya que puede ser obtenido sin receta médica en farmacias y herboristerías. En el ámbito sanitario se considera un fármaco inocuo, sin embargo está descrito en la literatura la aparición de complicaciones hemorrágicas intracraneales (hemorragias lobares, hematomas subdurales, hemorragias subaracnoideas y cerebelosas), un caso de hipema y dos casos de complicaciones hemorrágicas en el posoperatorio (4).

Los principales componentes del extracto de Ginkko biloba son los flavonoides y los terpenoides (ginkgolides y bilobalides). Los ginkgolides son antagonistas del receptor del factor activador de plaquetas (PAF), mecanismo que explicaría el potencial hemorrágico del Ginkgo biloba a través de su efecto inhibidor de la agregación plaquetaria (5); aunque hay estudios recientes que no han logrado evidenciar alteración de la función plaquetaria ni de la coagulación mediada por extractos de GB (6).

Este efecto hemorrágico puede estar potenciado al interactuar con otros fármacos con efecto antiagregante o anticoagulante como el ácido acetil salicílico, dicumarínicos y rofecoxib exacerbando las posibilidades de sangrado (7). Además estan descritas interacciones farmacológicas con el diltiazem y nicardipino.

Conviene recordar que sus supuestos beneficios en la preservación de la memoria o en el tratamiento de la demencia son objeto de controversia, existiendo el estudio clásico de Le Bars y cols. (8) y otros posteriores que demostraron mejoría cognitiva y de funcionamiento social en sujetos con demencia tratados con extracto de GB, y otros más recientes que no han demostrado mejoría cognitiva en ancianos sanos (9), ni en pacientes con "pérdida de memoria asociada a la edad" (AAMI) ni con demencia (10). Es decir, que en el momento actual no podemos considerarlo como un fármaco de alto valor terapéutico aunque se precisan de más estudios para poder realmente valorar su eficacia.

En resumen, el extracto de GB no es un fármaco totalmente inocuo, podría originar hemorragias intracraneales, podría interferir con episodios quirúrgicos y/o anestésicos y no tiene alto valor terapéutico lo que debería limitar su uso, al menos conjuntamente con fármacos antiagregantes o anticoagulantes, en la actualidad.

\section{F. J. Castellote Varona , M. P. Atienza Morales ${ }^{1}$}

Unidad de Valoración Geriátrica. Hospital Universitario Virgen de la Arrixaca. Murcia. ${ }^{1}$ Servicio de Análisis Clínicos. Hospital Comarcal de Hellín. Albacete

1. Instituto de Botánica. Universidad de Bonn. 2002. htpp://www.botanik.unibonn.de/conifers/gi/gi.

2. Sierpina VS, Wollschlaeger B, Blumenthal M. Ginkgo biloba. Am Fam Physician 2003; 68: 923-926.

3. Rowin J, Lewis SL. Spontaneous bilateral subdural hematomas associated with chronic Ginkgo biloba ingestion. Neurology. 1996; 46: 17751776.

4. Fessenden JM, Wittenborn W, Clarke L. Ginkgo biloba. A case report of herbal medicine and bleeding postoperatively from a laparoscopic cholecystectomy. Am Surg 2001; 67: 33-35.

5. Lamant V, Mauco G, Braquet P, Chap H, Douste-Blazy L. Inhibition of the metabolism of platelet activating factor (PAF-acether) by three specific antagonists from Ginkgo biloba. Biochem Pharmacol 1987; 36: 2749-2752.

6. Bal Dit Sollier C, Caplain H, Drouet L. No alteration in platelet function or coagulation induced by EGb761 in a controlled study. Clin Lab Haematol 2003; 25: 251-253.

7. De Blas B, Laredo LM, Vargas E. Interacciones de los fármacos más consumidos. In Ter Sist Nac Salud 2004; 28: 1-11.

8. Le Bars PL, Katz MM, Turan B, Alan AM. A placebo-controlled, double-blind, randomized trial of an extract of Ginkgo biloba for dementia. JAMA 1997; 278: 1327-1332.

9. Solomon PR, Adams F, Silver A, Zimmer J, De Veaux R. Ginkgo for memory enhancement: a randomized controlled trial. JAMA 2002; 288 : 835-840.

10. Van Dongen M, van Rossum E, Kessels A, Sielhorst H, Knipschil P. Ginkgo for elderly people with dementia and age-associated memory impairment: a randomized clinical trial. J Clin Epidemiol 2003; 56: 367-376. 


\section{Derrame pericárdico severo como primera manifestación de un carcinoma tímico}

\section{Sr. Director:}

El carcinoma tímico, es un tumor muy raro del timo, se caracteriza por la elevada frecuencia con que presenta invasión local y diseminación a distancia.

Como los timomas se origina en las células epiteliales del timo, pero en este caso las células manifiestan signos morfológicos claramente malignos, caracterizados por el alto grado de anaplasia y atipias. Con frecuencia falta la encapsulación y muy raramente van asociados a cuadros paraneoplásicos como la miastenia, que con cierta frecuencia se encuentra en los timomas (1).

En más de la mitad de los casos se trata de carcinomas indiferenciados, aunque comprenden también el carcinoma de células escamosas, el carcinoma linfoepitelioma like, el carcinoma de células del huso y el carcinoma de células claras (2).

Recientemente hemos tenido ocasión de diagnosticar un nuevo caso y nos parece interesante presentarlo brevemente dada su rareza.

Varón de 75 años de edad, con antecedentes personales de hipertensión arterial en tratamiento con amiloride, diabetes mellitus en tratamiento con acarbosa e hiperuricemia en tratamiento con alopurinol; implantación de marcapasos definitivo hacía 3 años por bradicardia sintomática.

Consultó por un cuadro de dolor torácico y disnea de unas semanas de evolución, siendo ingresado en Cardiología, donde se objetivo en el ecocardiograma un derrame pericárdico severo. Posteriormente se solicitó una TAC torácica en la que se observó una masa en el mediastino anterior con calcificaciones en su interior, sugestiva de carcinoma tímico, teratoma o menos probablemente linfoma (Fig. 1).

Hemograma, la hemostasia y la bioquímica fueron normales, salvo una glucemia de $126 \mathrm{mg} / \mathrm{dl}$.

Fue remitido al Hospital Gregorio Marañón de Madrid, donde se realizó una ventana pleuropericárdica mediante toracotomía anterior izquierda, obteniéndose además una biopsia del pericardio y de la masa mediastínica. La anatomía patológica fue informada como timoma de células fusiformes (tipo A medular).

Posteriormente fue remitido al Servicio de Cirugía Cardiaca del mismo hospital para extirpación de la mayor cantidad posible de tejido tumoral. Se realizó resección de la masa tímica,

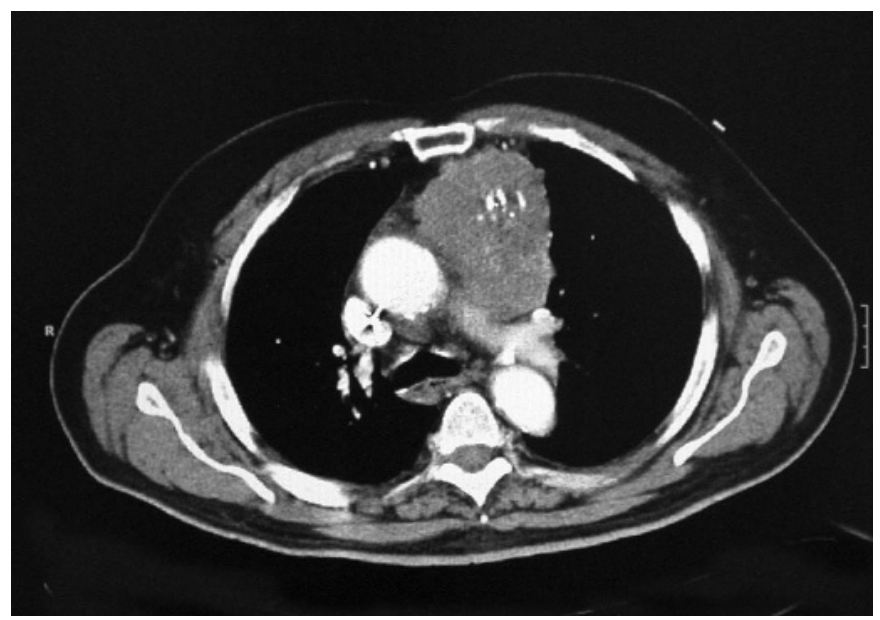

Fig. 1. Masa en el mediastino anterior con calcificaciones en su interior, sugestiva de carcinoma tímico, teratoma o linfoma. que infiltraba la adventicia aórtica y pulmonar, así como la pleura izquierda y el lóbulo superior izquierdo pulmonar. Se efectúo asimismo una pericardiectomía y una resección en cuña del lóbulo superior izquierdo, se biopsiaron adenopatías aortopulmonares, que se encontraron libres de enfermedad. El informe definitivo de la anatomía patológica fue de carcinoma tímico, pericarditis fibrosa inespecífica y linfadenitis reactiva inespecífica.

Una TAC de tórax realizada quince días después mostró en el mediatino anterior la persistencia de una masa hipodensa de 3,9 x 3,4 centímetros, compatible con masa residual postquirúrgica, no existía derrame pericárdico. Se procedió a realizar tratamiento de radioterapia, con respuesta completa.

Se ha clasificado el carcinoma tímico, como de alto grado, que comprende el carcinoma tímico linfoepitelioma-like, carcinoma de célula pequeña, carcinoma anaplásico de célula grande, carcinoma sarcomatoide, carcinoma de células claras y de bajo grado que comprende el carcinoma escamoso queratinizante, carcinoma escamoso basalioide y el carcinoma mucoepidermoide (3).

Los carcinomas del grupo de bajo grado presentan una supervivencia mediana en torno de un año, siendo la mitad en los de alto grado. Menos de la mitad de los casos se encuentran en estadios localizados, en un $20 \%$ se encuentran metástasis (1). Frecuentemente se presentan con afectación pleural o pulmonar, también los ganglios mediastínicos, cervicales, el hígado y los huesos pueden encontrarse afectados (1). Se han encontrado datos de infección previa con el virus de Epstein-Barr, especialmente en el subtipo linfoepitelioma-like en pacientes jóvenes (4). Se ha encontrado una expresión más frecuente de p53 y del bcl-2 en el carcinoma tímico que en el timoma, fundamentalmente en los casos de alto grado (5).

Con respecto al tratamiento, se debe intentar realizar una resección completa, aunque habitualmente como en el caso que hemos presentado no es posible y suele persistir tumor residual. La aplicación de radioterapia postoperatoria puede aumentar la supervivencia (6).

Los carcinomas tímicos son mucho menos sensibles a la quimioterapia que los timomas; se han empleado combinaciones con cisplatino, VP-16, bleomicina e ifofosfamida con resultados variables. La duración media de la respuesta oscila alrededor del año y la supervivencia media sobrepasa ligeramente los dos años y medio (7-9).

F. Marcos Sánchez, A. Vázquez García ${ }^{1}$, A. González Pinto², I. Alto Castaño, A. Viana Alonso

Servicios de Medicina Interna $y{ }^{l}$ Cardiología, Hospital Nuestra Señora del Prado. Talavera de la Reina, Toledo. ${ }^{2}$ Servicio de Cirugía Cardiaca. Hospital Gregorio Marañón. Madrid

1. Castellanos Diez J, Lázaro Quintela M, Jorge Fernández M, Carrasco Álvarez JA, Pérez-López E. Timoma y carcinoma tímico. En: Guía práctica de tumores poco frecuentes. Lázaro Quintela M, Jorge Fernández M, Carrasco Álvarez J, Castellanos Diez J. Madrid: Editorial You\&Us, SA, 2003. p. 23-35.

2. Snover DC, Levine GD, Rosai J. Thymic carcinoma: five distinct histological variants. A, J Surg Pathol 1982; 6: 451.

3. Daugaard G, Hansen H, Roth M. Combination chemotherapy for malignant thymomas. Ann Intern Med 1983; 99: 189-190.

4. Wu TC, Kuo TT. Study of Epstein-Barr virus early RNA 1 (EBER 1) expression by in situ hybridization in thymic epithelial tumors of Chinese patients in Taiwan. Hum Pathol 1993; 24: 235-238.

5. Kuo TT, Chan JK. Thymic carcinoma arising in thymoma is associated with alterations in immunohistochemical profile. Am J Surg Pathol 1998; 22: $1474-1481$ 
6. Sunpaweravong P, Kelli K. Treatment of Thymona: a comparative study between Thailand and the United States and a review of the literature. Am J Clin Oncol 2004; 27: 236-246.

7. William J, Quantin X, Valette S, Khial F, Pujol JL. Multimodality treatment program in invasive thymic epithelial tumor. Am J Clin Oncol 2005; $28: 5-7$.

8. Loehrer PJ, Jiroutek M, Aisner S, Aisner J, Green M, Thomas CR, et al. Combined etoposide, ifosfamide and cisplatin in the treatment of patients with advanced thymoma and thymic carcinoma. An Intergroup trial. Cancer 2001; 91: 2010-2015.

9. Blossom GB, Steiger Z, Stephenson LW. Carcinoma del timo. Neoplasias del mediastino. Cáncer: principios y práctica de oncología. De Vita Jr VT, Hellman S, Rosenberg SA. Madrid: Ed. Panamericana-Aran, 2000. p. 960.

\section{Infección nosohusial por Pseudomonas putida}

\section{Sr. Director:}

Graham (1-3) definió la infección nosohusial como aquella adquirida por un paciente en el ámbito extrahospitalario relacionada con los tratamientos, generalmente antibióticos intravenosos, administrados en el domicilio. Su idea era la de definir un tipo de infección con similitudes y a al vez diferencias con la infección nosocomial. El término derivaría de la raíz griega vódo ‘ (nosos) o enfermedad y de la palabra hus del inglés antiguo, y del que deriva el término "house", casa. Otros autores han denominado a estas infecciones nosodomiales, utilizando la raíz griega y la latina domus, aunque en las escasas referencias existentes en la literatura médica, ninguna en nuestro país en nuestro conocimiento, se utiliza el término de infección nosohusial $(4,5)$.

$P$. putida es un bacilo gram negativo, aerobio que se puede aislar tanto en el suelo como en el agua o como espécimen clínico y que en ocasiones se comporta como patógeno oportunista.

Presentamos el caso de un varón de 50 años con antecedente de traumatismo con fractura abierta de tibia y peroné de pierna izquierda en 1982 y diagnosticado en la actualidad de osteomielitis de tibia izquierda, motivo de su ingreso hospitalario. Tras realizar tratamiento con una limpieza quirúrgica, donde se recogieron muestras para cultivo en las que se aisló $S$. aureus sensible a la cloxacilina, se inició antibioterapia endovenosa con cloxacilina $12 \mathrm{~g} / \mathrm{d}$ más gentamicina $240 \mathrm{mg} / \mathrm{d}$, presentando el paciente evolución favorable. Por ello se decidió trasladar el paciente a la Unidad de Hospitalización a Domicilio, tras acceder a una vía venosa central en subclavia derecha, para completar el tratamiento antibiótico parenteral en su domicilio. A las tres semanas de estancia en el domicilio el paciente presentó de manera brusca un episodio de tiritona y fiebre elevada $\left(38,5^{\circ} \mathrm{C}\right)$, sin focalidad. Se decidió inicialmente mantener el catéter, no había signos locales de infección, y se tomaron diversas muestras para cultivo pero, tras recibir el informe de microbiología del crecimiento de un BGN en el hemocultivo tomado a través del catéter venoso central, 48 h más tarde se retiró el catéter venoso central y se envió la punta para cultivo. Ambas muestras fueron finalmente informadas como positivas para Pseudomonas putida (sistema api 20 NE de BioMerieux S.A.). Los cultivos de las conexiones y del contenido de la bolsa de infusión, que contenía la cloxacilina, fueron negativos. Con el juicio clínico de bacteriemia relacionada con catéter venoso central se inició un ciclo antibiótico según resultado del antibiograma con la asociación de tobramicina $300 \mathrm{mg} / \mathrm{d}$ endovenosa y ciprofloxacino oral $750 \mathrm{mg} / 12 \mathrm{~h}$, con evolución favorable, sin requerir de ingreso hospitalario.

Según aumenta el número de pacientes tratados en sus domicilios con antibióticos parenterales y con accesos venosos centrales o periféricos, aumentan las posibilidades de aparición de complicaciones derivadas de su utilización, siendo las infecciones de catéter, como en el hospital aunque en menor medida (5), uno de los mayores problemas. Los pacientes se van asemejando más a los ingresados en los hospitales tanto en su situación general como en los medios utilizados en su tratamiento aunque con las diferencia de ser el "único paciente ingresado en ese lugar" y por ello minimizar el riesgo de infección o colonización a otro o de otro. Estas infecciones nosohusiales o nosodomiales (pensamos que este término es más correcto) aparecen entre un 1-2\% (6) de los pacientes, según un registro internacional de casos de tratamientos antibióticos parenterales extrahospitalarios y aun hoy son poco conocidas y estudiadas. A pesar de ello su proporción se estima inferior al de las infecciones nosocomiales (6). En cualquier caso hay que tener en cuenta que con los tratamientos realizados actualmente en los domicilios, antibióticos parenterales, nutriciones parenterales, hemodiálisis ambulatoria, etc., es esperable que cambie el espectro de patógenos que infecte al "paciente de la comunidad" con las consiguientes implicaciones diagnósticas y terapéuticas, como ya han sugerido algunos autores (7). En el caso presentado, el paciente fue diagnosticado de una bacteriemia relacionada con el catéter, vía central, por un microorganismo poco común $P$. putida. Este microorganismo ha sido involucrada en bacteriemias en neonatos (8), y en una serie de 55 casos (9) en infecciones del tracto urinario, neumonía, sepsis, infección de herida, meningitis y peritonitis pero sigue siendo considerado como un patógeno raro. Aunque la infección apareció en la tercera semana tras el alta hospitalaria, no creemos que el origen de la misma fuera hospitalario, de forma anecdótica, el pasado año, el Ministerio de Sanidad y Consumo ordenó la retirada del mercado de determinados lotes de jabón líquido, de una conocida marca comercial, al haberse detectado contaminación bacteriana de este producto por $P$. putida y $P$. fluorescens. Aunque nuestro paciente no había utilizado dicho jabón, no podemos descartar que el microorganismo pueda encontrarse en el ámbito doméstico.

Con esta comunicación pretendemos alertar sobre la existencia de las infecciones nosodomiales o nosohusiales y hacer hincapié en la necesidad de controles estrictos en los pacientes sometidos a tratamientos endovenosos en los domicilios.

\section{A. Goenaga Sánchez, M. Millet Sampedro, J. A. Carrera Macazaga, C. Garde Orbáiz}

Unidad de Hospitalización a Domicilio. Hospital Donostia. San Sebastián. Guipúzcoa

1. Graham DR, Keldermans MM, Klemm LW, Semenza NJ, Shafer ML. Infectious complications among patients receiving home intravenous therapy with peripheral, central or peripherally placed central venous catheters. Am J Med 1991; 91 (3B): 95S-100S.

2. Graham DR. Nosohusial infections: complications of home infusion therapy. Infect Dis Clin Pract 1993; 2: 158-161.

3. Graham, DR. Nosohusial infections: a decade of surveillance for complications of home intravenous therapy. Infect Control Hosp Epidemiol 2000; 21(2): 152 .

4. Tice AD. Handbook of outpatient parenteral therapy for infectious diseases. 1st Ed. New York: Scientific American, INC, 1997.

5. Tokars JI, Cookson ST, McArthur MA, Boyer CL, McGeer AJ. Jarvis WR. Prospective evaluation of risk factors for bloodstream infection in patients receiving home infusion therapy. Ann Intern Med 1999; 131: 340-7.

6. Tice AD, Nolet BR. Update on Outpatient Parenteral Antimicrobial Therapy. Home Health Care Consultant 2001; 8: 22-29.

7. Friedman ND, Kaye KS, Stout JE, McGarry SA, Trivette SL, Briggs JP, et al. Health care-associated bloodstream infections in adults: a reason to change the accepted definition of community-acquired infections. Ann Intern Med 2002; 137: 791-797. 
8. Bouallegue O, Mzoughi R, Weill FX, Mahdhaoui N, Ben Salem Y, Sboui $\mathrm{H}$, Grimont F, Grimont PA. Outbreak of pseudomonas putida bacteraemia in a neonatal intensive care unit. J Hosp Infect 2004; 57 (1): 88-91.

9. Yang CH, Young T, Peng MY, Weng MC. Clinical spectrum of Pseudomonas putida infection. J Formos Med Assoc 1996; 95 (10): 754-61.

\section{Absceso subfrénico izquierdo primario por Streptococcus intermedius}

\section{Sr. Director:}

Streptococcus milleri, también denominado Streptococcus intermedius, es un grupo de Estreptococo viridans constituido por las especies $S$. intermedius, $S$. constellatus y $S$. anginosus (1). Aunque son comensales habituales de la cavidad oral, orofaringe, tracto gastrointestinal y genital, pueden ocasionar infecciones piógenas e invasivas, con tendencia a la formación de abscesos, posibilidad de bacteriemia, endocarditis, y ocasionalmente septicemia y shock séptico.

Se presenta el caso de un varón de 60 años, con diabetes mellitus tipo 2 en tratamiento con repaglinida $(1 \mathrm{mg} /$ día $)$, fumador de 20 cigarrillos/día e ingesta etílica de 60 g/día. Refería cuadro de 15 días de evolución con dolor en epigastrio-hemiabdomen superior, hiporexia y sensación de plenitud gástrica, sin vómitos y sin alteración del hábito intestinal, sin fiebre ni sensación distérmica. No refería disnea, tos o dolor torácico. En exploración física destacaba: taquicardia (100lpm), palidez cutánea, dolor y sensación de empastamiento en zona epigástrica, sin peritonismo. En analítica destacaban, Bioquímica: hiperglucemia (382 mg/dL), hiponatremia ligera $(133 \mathrm{mEq} / \mathrm{L})$, siendo normales amilasemia, lipasemia y bioquímica hepática. Hemograma: leucocitosis (17.700 $\left./ \mathrm{mm}^{3}\right)$ con neutrofilia $(90 \%$ segmentados), ligera anemia $(\mathrm{Hb}$ 12,9 g/dL; Hto $36 \%$ ), normocroma y normocítica. Velocidad de sedimentación globular elevada $\left(98 \mathrm{~mm} / 1^{\mathrm{a}}\right.$ hora). Orina: glucosuria, cetonuria y proteinuria, con sedimento normal y urocultivo negativo. Otras determinaciones analíticas (hormonas tiroideas, proteinograma, marcadores tumorales CEA y CA 19-9) fueron normales. En radiología de tórax se detectó derrame pleural izquierdo, realizándose toracocentesis diagnóstica con obtención de líquido pleural tipo exudado con glucosa $135 \mathrm{mg} / \mathrm{dL}$ (glucemia $156 \mathrm{mg} / \mathrm{dL}$ ), proteínas 3,9 g/dL (en suero 6,2 g/dL), LDH $240 \mathrm{UI} / \mathrm{L}$ (en suero $539 \mathrm{UI} / \mathrm{L}$ ), 850 leucocitos $/ \mathrm{mm}^{3}$ (85\% neutrófilos), pH 7,43; amilasa < 30 UI/L, ADA 17 UI/L; sin detección de microorganismos en tinciones Gramm, Ziehl y en cultivos aerobios, anaerobios y de micobacterias, y con citología negativa para malignidad. En ecografía abdominal, hígado y vías biliares eran normales, sin detectarse alteraciones relevantes en el resto de exploración. En tomografía computarizada (TC) torácica destacaba derrame pleural izquierdo con atelectasia secundaria del lóbulo inferior izquierdo (LII). En TC abdominal (Fig. 1) se detectó colección líquida subfrénica izquierda perigástrica (A), que se drenó percutaneamente, obteniéndose $400 \mathrm{ml}$ de líquido purulento en el que se aisló Streptococcus intermedius multisensible. Se pautó inicialmente cefotaxima ( $1 \mathrm{~g} / 8$ horas/i.v.) y luego levofloxacino oral ( $500 \mathrm{mg} /$ día) que se mantuvo hasta completar 1 mes de tratamiento, con buena evolución clínica y resolución del absceso subfrénico izquierdo, así como del derrame pleural (y atelectasia secundaria LII). Se completó estudio con endoscopia oral que no mostró hallazgos relevantes; ecocardiografía transto-

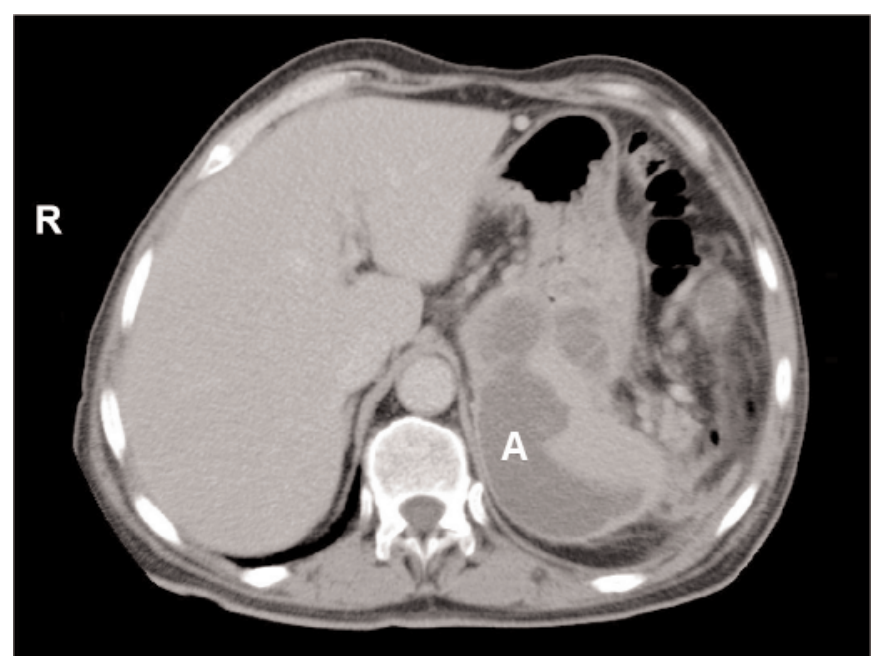

Fig. 1.

rácica y transesofágica, sin detectar vegetaciones valvulares ni otras alteraciones significativas, y estudio esófago-gastroduodenal con contraste hidrosoluble, que no mostró trayectos fistulosos desde cavidad gástrica.

Las infecciones del grupo $S$. milleri se localizan preferentemente en abdomen (abscesos hepáticos y subfrénicos, colangitiscolecistitis, apendicitis), cabeza y cuello, tórax, sistema nervioso central, así como endocarditis y bacteriemia (2-4). Son más frecuentes en pacientes con diabetes mellitus, cáncer (carcinomas, leucemias), drogadicción intravenosa o neutropenia, pudiendo ser la forma de presentación de una neoplasia subyacente $(5,6)$. La especie $S$. intermedius es la menos frecuentemente aislada del grupo $S$. milleri, y la que tiene mayor propensión a la formación de abscesos, preferentemente hepáticos, cerebrales y en cabeza y cuello (7). Los abscesos subfrénicos izquierdos son habitualmente secundarios a complicaciones postoperatorias abdominales, principalmente tras cirugía gástrica, pancreática o esplenectomía (8). Con mucha menor frecuencia son primarios o de aparición espontánea, como el caso aquí presentado.

Las infecciones del grupo $S$. milleri suelen responder bien a penicilina $\mathrm{G}$ y cefalosporinas, aunque se registra un aumento progresivo de la concentración mínima inhibitoria (CMI) frente a beta-lactámicos (9), recomendándose iniciar tratamiento con penicilina $\mathrm{G}$ y gentamicina. También pueden emplearse cefalosporinas de $3^{\mathrm{a}}$ o $4^{\mathrm{a}}$ generación (cefotaxima, ceftriaxona, cefepima), especialmente en cuadros bacteriémicos. En alérgicos a penicilina pueden emplearse vancomicina y clindamicina. El drenaje de los abscesos (percutáneo o quirúrgico), casi siempre es necesario.

E. Crespo Valadés, J. M. Fernández Blanco, J. Troya García, M. Malmierca Corral

Servicio de Medicina Interna. Hospital Virgen de la Salud. Toledo

1. Antony SJ, Stratton CW. Streptococcus intermedius group. En: Mandell GL, Bennet JE, Dolin R, eds. Principles and practice of infectious diseases. $5^{\text {a }}$ ed. Philadelphia: Churchill Livingstone, 2000; 2183-9.

2. Bert F, Bariou-Lancelin M, Lambert-Zechovsky N. Clinical significance of bacteremia involving the Streptococcus milleri group: 51 cases and review. Clin Infect Dis 1998; 27: 385-7.

3. Crespo Valadés E, Barberá Farré, Ruiz de Gauna Martín E, Cabra Dueñas J. Bacteriemia, endocarditis y absceso cervical por Streptococcus intermedius. An Med Interna (Madrid) 2003; 20: 601-2. 
4. Karchmer AW, Torchiana DF, Chae CU, Afridi NA, Houser SL. Case 29-2004: A 75-year-old woman with acute onset of chest pain followed by fever. N Eng1 J Med 2004; 351; 12040-8.

5. Conde Martel A, Martín Pérez P, Suárez Ortega S, Bordes Benítez A. Abscesos hepáticos por Streptococcus intermedius como primera manifestación de un carcinoma renal. Rev Esp Enferm Dig 2002; 94: 45-6.

6. Tzur T, Liberman S, Felzenstein I, Cohen R, Rivkind AI, Almogy G. Liver abscesses caused by Streptococcus milleri: an uncommon presenting sign of silent colonic cancer. Isr Med Assoc J 2003; 5: 206-7.

7. Clarridge JE, Attorri S, Musher DM, Herbert J, Dunbar S. Streptococcus intermedius, Streptococcus constellatus, and Streptococcus anginosus ("Streptococcus milleri group") are of different clinical importance and are not equally associated with abscess. Clin Infect Dis 2001; 32: 1511-5.

8. Patterson HC. Left subphrenic abscesses. Am Surg 1977; 43: 430-3.

9. Bantar C, Fernández Canigia L, Relloso S, Lanza A, Bianchini H, Smayevsky J. Species belonging to the "Streptococcus milleri" group: antimicrobial susceptibility and comparative prevalence in significant clinical specimens. J Clin Microbiol 1996, 34: 2020-2.

\section{Peritonitis bacteriana espontánea por Listeria monocytogenes. A propósito de un nuevo caso}

\section{Sr. Director:}

La peritonitis bacteriana espontánea (PBE) es una complicación grave que aparece habitualmente en pacientes con cirrosis hepática en estadio avanzado. Los microorganismos más frecuentemente implicados son enterobacterias procedentes del tubo digestivo, sobre todo Escherichia coli y Klebsiella spp (1). Listeria monocytogenes es una causa muy infrecuente de PBE, habiéndose comunicado en la literatura 35 casos hasta la fecha (2-7). Presentamos un nuevo caso de PBE por Listeria monocytogenes ocurrido en nuestro hospital en un enfermo con cirrosis hepática.

Se trata de un paciente de 71 años con antecedentes de cirrosis hepática de origen etílico en estadio B (8 puntos) según la clasificación de Child-Pugh, con episodios previos de descompensación hidrópica y hemorragia digestiva alta por varices esofágicas. No estaba siendo tratado con antibióticos profilácticos para la PBE. Acude a urgencias por malestar general, melenas y dolor abdominal difuso de 4 días de evolución sin fiebre. A la exploración física se encontraba consciente y afebril y se observaron estigmas de hepatopatía crónica. El abdomen era doloroso a la palpación con aumento del perímetro abdominal sugestivo de ascitis franca. En el tacto rectal se apreciaron restos de deposiciones melénicas. Al ingreso presentaba los siguientes datos analíticos: 11.000 leucocitos $\mathrm{mm}^{3}$ ( $80 \%$ neutrófilos, $11 \%$ linfocitos, $8 \%$ monocitos), Hb 7,5 g/dL, Hcto 21,6\%, VCM: 95,1 fL, plaquetas $229.000 / \mathrm{mm}^{3}$, actividad de protrombina $54 \%$, creatinina 1.4 $\mathrm{mg} / \mathrm{dL}$, bilirrubina total $2.3 \mathrm{mg} / \mathrm{dL}$, GOT $60 \mathrm{UI} / \mathrm{L}$ y GPT 42 UI/L. Se efectuó una gastroscopia que demostró la presencia de varices esofágicas en grado II sin signos de sangrado reciente. Se practicó una paracentesis diagnóstica obteniéndose un líquido ascítico (LA) claro con 600 leucocitos/mL (330 polimorfonucleares), $145 \mathrm{mg} / \mathrm{dL}$ de glucosa y $1 \mathrm{~g} / \mathrm{dL}$ de proteínas totales. Con el diagnóstico de PBE se inició tratamiento antibiótico empírico con ciprofloxacino por vía intravenosa $(400 \mathrm{mg} / 12 \mathrm{~h})$. A las 48 horas del ingreso se obtuvo crecimiento microbiano en el LA por el sistema automático Bact/Alert 3D (bioMérieux) para cultivos en medio líquido, observándose en la tinción de Gram del caldo cocobacilos grampositivos de tipo corineforme. Ante la sospecha microbiológica de que pudiera ser Listeria spp se añadió al tratamiento ampicilina $(1 \mathrm{~g} / 6 \mathrm{~h})$ por vía intravenosa. El microorganismo fue identificado posteriormente como Listeria monocytogenes mediante el sistema comercial API Coryne ${ }^{\circledR}$ (bioMérieux). El hemocultivo fue negativo. La respuesta clínica fue satisfactoria con esterilización del LA tras 15 días de tratamiento antibiótico. Durante su evolución posterior, el paciente sufrió una hemorragia digestiva por rotura de varices esofágicas que precisó de ligadura endoscópica, además de un empeoramiento progresivo de la función renal con aparición de encefalopatía en grado IV, falleciendo a los 20 días del ingreso.

Listeria monocytogenes es un cocobacilo grampositivo no esporulado y anaerobio facultativo, que habitualmente produce infecciones graves en inmunodeprimidos y en inmunocompetentes en edades extremas de la vida. Las manifestaciones clínicas más frecuentes de la listeriosis son la bacteriemia y la meningitis, pero excepcionalmente se han descrito casos de neumonía, endoftalmitis, artritis séptica, endocarditis, osteomielitis y peritonitis (8). La PBE por Listeria monocytogenes es más frecuente en enfermos con cirrosis hepática pero también puede aparecer en pacientes con neoplasias, diálisis peritoneal y trasplantados hepáticos (2). Resulta muy llamativo que gran parte de los casos publicados lo hayan sido por autores españoles. Con nuestro caso, son 28 los pacientes cirróticos con PBE por Listeria monocytogenes comunicados $(2,3,6,7)$ y, de éstos, 19 (68\%) son españoles $(2,6,7)$. La razón de este predominio en nuestro país es desconocida, pero se ha relacionado con la mayor incidencia que presenta la listeriosis en España en relación con factores dietéticos (mayor consumo de frutas y vegetales), climáticos y demográficos (9). La patogenia de esta enfermedad no se conoce bien pero es muy probable que Listeria monocytogenes provenga del tubo digestivo. De hecho, este microorganismo está presente en el tracto digestivo del 14\% de los individuos sanos (10) y se sospecha que este porcentaje puede ser todavía mayor en enfermos cirróticos tratados con antibióticos profilácticos (6). Listeria monocytogenes podría alcanzar el LA por translocación bacteriana desde el tubo digestivo a través de los folículos de Peyer o por diseminación hematógena. La infección se vería favorecida por la presencia de alteraciones en el sistema reticuloendotelial y por la disminución de la capacidad opsonizante y bactericida de las proteínas del LA (11). Las manifestaciones clínicas de la PBE por Listeria monocytogenes son superponibles a las producidas por otros microorganismos, salvo que en ocasiones puede haber otros focos de infección, especialmente meningoencefalitis concomitante $(9,12)$.

La realización de un diagnóstico microbiológico correcto es fundamental puesto que Listeria monocytogenes es resistente a cefotaxima, que constituye el tratamiento antibiótico empírico recomendado cuando existe sospecha de PBE (1). El tratamiento específico se basa en el uso de ampicilina asociada o no a un aminoglucósido, si bien estos últimos deben restringirse en los enfermos cirróticos por el riesgo de nefrotoxicidad. Como alternativa, algunos autores han utilizado con éxito el cotrimoxazol (11). Dada la rareza de este proceso, no está perfectamente definida la duración del tratamiento, pero se recomienda mantenerlo hasta que se resuelva la ascitis y se produzca la esterilización del cultivo del LA y del hemocultivo (12). La mortalidad puede oscilar entre el $17-28,5 \%(9,12)$.

En resumen, aportamos un caso más a los ya publicados de PBE por Listeria monocytogenes. Este microorganismo, a pesar de su infrecuencia, es responsable de un número no despreciable de PBE en nuestro país y por ello debe ser tenido en cuenta como posible agente causal de este proceso, especialmente en enfermos cirróticos que no evolucionan bien con el tratamiento antibiótico 
empírico inicial. Dado que Listeria monocytogenes es resistente a cefotaxima, el diagnóstico microbiológico resulta esencial para el correcto manejo terapéutico de la infección.

\section{Tena Gómez, R. Carranza González, M. R. Burón Fernán- dez ${ }^{1}$, F. Pérez-Roldán ${ }^{2}$}

Laboratorio de Microbiología. 'Servicio de Medicina Interna. ${ }^{2}$ Sección de Digestivo. Hospital General La Mancha Centro. Alcázar de San Juan. Ciudad Real

1. Levison ME, Bush LM. Peritonitis and other intra-abdominal infections. In: Mandell GL, Bennett JE, Dolin R, editors. Principles and Practice of Infectious Diseases. $5^{\mathrm{a}}$ ed. Philadelphia: Churchill Livingstone; 2000, p. 821-56.

2. Jorquera F, Espinel J, Fernández MJ, Muñoz F, Herrera A, Vivas S, et al. Peritonitis bacteriana espontánea por Listeria monocytogenes. Gastroenterol Hepatol 1998; 21: 489-91.

3. Jayaraj K, Di Bisceglie AM, Gibson S. Spontaneous bacterial peritonitis caused by infection with Listeria monocytogenes: a case report and review of the literature. Am J Gastroenterol 1998; 93: 1556-8.

4. Adeonigbagbe O, Khademi A, Karowe M, Gualtieri N, Robilotti J. Listeria monocytogenes peritonitis. An unusual presentation and review of the literature. J Clin Gastroenterol 2000; 30: 436-7.

5. Rohde H, Horstkotte MA, Sobottka I, Klose H, Mack D. Spontaneous bacterial peritonitis due to Listeria monocytogenes in a patient with primary pulmonary hypertension. Eur J Clin Microbiol Infect Dis 2002; 21: 323-5.

6. Uriarte I, Bujanda L, Peña P. Peritonitis bacteriana espontánea por Listeria monocytogenes. An Med Interna 2002; 19: 214-5.

7. Casado M, Torres M, Gálvez C, Rodríguez-Maresca M, Sánchez-Yebra W, Vázquez JA. Peritonitis bacteriana espontánea por Listeria monocytogenes. Enferm Infecc Microbiol Clin 2002; 20: 43-4.

8. Lorber B. Listeriosis. Clin Infect Dis 1997; 24: 1-9.

9. Vázquez J, Such J, Ena J, Argüello L, Ortiz de la Tabla V, Mingot M, et al. Peritonitis bacteriana espontánea por Listeria monocytogenes. Rev Esp Enf Digest 1995; 87; 407-11.

10. Gómez-Mampaso E, Teruel JL, Cascales P, García-Martín F, Baquero F, Ortuño J. Portadores fecales de Listeria y trasplante renal. Rev Esp Microbiol Clin 1987; 2: 99-101.

11. Fernández-Solá A, Pigrau C, Almirante B, Gasser I. Peritonitis bacteriana espontánea por Listeria monocytogenes. Med Clin (Barc) 1995; 104 : 199.

12. Pérez-Roldán F, Bañares R, Piqueras B, De Diego A, Castellote I, Casado M, et al. Peritonitis bacteriana espontánea por Listeria monocytogenes. Rev Esp Enf Digest 1995; 87: 889-92.

\section{Hidroneumotórax secundario a colocación de sonda nasogástrica}

\section{Sr. Director:}

La alimentación enteral utilizando sondas flexibles nasogástricas es cada vez más utilizada en pacientes críticos y malnutridos. Aunque más segura que la nutrición parenteral, no está exenta de complicaciones. Se incluyen la perforación esofágica y la penetración en pulmón causando neumotórax e infusión de la alimentación en pulmón y espacio pleural. Escasamente comunicadas por su rareza la mayoría de las complicaciones broncopleurales están en relación con la colocación de la sonda en bronquio principal derecho y suelen ocurrir en pacientes obnubilados o intubados. Presentamos el caso de un paciente que presenta hidroneumotórax tras colocación de sonda nasogástria inadvertido hasta 24 horas después cuando la presencia de fiebre condujo a la realización de una radiografía de tórax.

Hombre de 61 años, con antecedentes de etilismo quien a consecuencia de politraumatismo es traído a urgencias presentándose desorientado y somnoliento. En la exploración física destacaba un Glasgow GCS de 13. 24 respiraciones/minuto, temperatura $36,5^{\circ} \mathrm{C}$, En la auscultación pulmonar roncus bilaterales. En la analítica destacaba: hemoglobina, $10 \mathrm{~g} / \mathrm{dl}$; hematocrito, 30\%; y resto de bioquímica normal. TAC craneal: fractura frontoparietal derecha. Pequeño hematoma epidural, temporal derecho, con contusión hemorrágica intraparenquimatosa. La radiografía de tórax no evidenció anomalías relevantes. Gasometría: pH. 7,40; $\mathrm{pCO}_{2}: 30 ; \mathrm{pO}_{2} 72 \mathrm{mmHg}$. $\mathrm{Al} 3^{\circ}$ día de estancia en Servicio de Reanimación presenta deterioro de nivel de conciencia y precisa intubación y ventilación mecánica. Se realizó traqueostomía percutánea y a los x días desconexión del respirador. Se traslada a planta de neurocirugía para continuar rehabilitación. Se reintroduce sonda nasogástrica (Flexiflo 12F) que el paciente se había retirado, para continuar la nutrición enteral. A las 6 horas presenta febrícula y sudoración. La persistencia del cuadro con temperatura de $38^{\circ}$ condujo a realizar una radiografía de tórax a las 24 horas que mostró la mala posición de la sonda (Fig. 1) y opacificación de pulmón derecho interpretado como hidroneumtórax, que se confirmó con tomografía computerizada de tórax. Se procedió a colocar tobe de tórax ( $\mathrm{n}^{\circ}$ 24) drenando en total $1900 \mathrm{cc}$ de las mismas caractrerísticas que el preparado de nutrición (Merite$\left.n \mathrm{e}^{\varpi}\right)$.Se instauró tratamiento antibiótico con ceftacidima y clindamicina. Se realizó una fibrobroncosciopia a través de la traqueostomía visualizando y aspirando, cantidad importante del mismo contenido alimenticio, que ocupaba todo el árbol bronquial derecho. La evolución posterior desde punto de vista respiratorio fue buena. Para continuar el tratamiento nutricional se practicó una gastrostomía enteroscópica percutánea (PEG).

El derrame pleural iatrogénico representa un porcentaje nada despreciable de todos los derrames pleurales. Puede ser secundario a fármacos, radioterapia, escleroterapia endoscópica, sobrecarga de fluidos, postcirugía de by-pass coronario, aortografía translumbar, desplazamiento de catéteres en vías centrales y de sondas de alimentación enteral entre otros (1).

La tasa de inserción de sondas nasogástricas y nasoentéricas en la tráquea y árboles bronquiales varía entre 0,3 y $15 \%$. Si la penetración de la via aérea y la introducción de alimento ocurren sin reconocerse

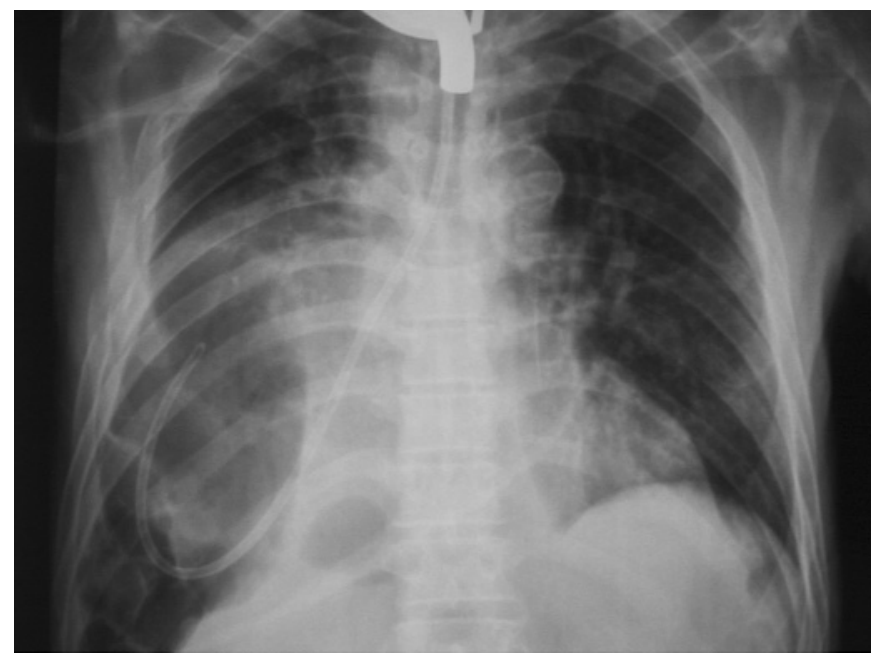

Fig. 1. Radiografía de tórax, donde se observa la posición de la sonda nasogástrica en cavidad pleural. 
pueden conducir a una neumonitis y ser fatal. Por otra parte la penetración en la vía aérea puede causar neumotórax, sobre 100 casos reportados y en uno de ellos como en nuestro caso, se había identificado aspirando la alimentación mediante toracocentesis (2).

El aumento de la importancia que se le da a la nutrición para un favorable evolución de cualquier patología conduce a un mayor uso de sondas de alimentación enteral. La nutrición enteral es además fácil, barata, confortable y con menos complicaciones que la parenteral y actualmente se utilizan sondas más blandas y flexibles que irritan minimamente y pueden mantenerse hasta 6 semanas. Los estiletes usados para facilitar la inserción y avance de la sonda, al aumentar la rigidez del tubo pueden también provocar mayo riesgo de perforación de estructuras. Entre los factores de riesgo para presentar complicaciones pleuropulmonares están la disminución del nivel de conciencia, reciente intubación endotraqueal, abolición de reflejo nauseoso, uso de bloqueantes neuromusculates y la existencia de estrictura esofágica (3).

La maniobra convencional para confirmar la correcta localización de la sonda consiste en insuflar aire seguida de la auscultación sobre el cuadrante superior izquierdo (el característico sonido del aire entrando en el estómago indica la colocación gástrica) o la aspiración del fluido. Sin embargo dicha maniobra puede no detectar una inserción inadecuada de la sonda en la vía aérea, por lo que creemos como otros autores que es recomendable realizar una radiografía de tórax antes de utilizar la misma (4). En algunas ocasiones ni siquiera la radiografía identifica la mala posición de la sonda, así Hendry et al (5) hallaron que 11 pacientes con sonda mal posicionada y con radiografía previa, que esta no identificaba la mal posición en 3 pacientes (27\%) por lo que algunos autores recomiendan realizar capnografía (5), laringoscopia o broncoscopia para asegurar la entrada en esófago de la sonda, técnicas que no son de rutina en nuestra práctica clínica (2).

\section{J. J. Blanco-Pérez, M. Barreiro Torres', J. Tábara²}

Servicios de Neumología, 'Anestesia y Reanimación y ${ }^{2}$ Neumología. Complexo Hospitalario Ourense. Ourense

1. Light RW. Pleural effusion due to miscellaneous diseases. In: Willians and wilkins editors. Pleural diseases. 3rd ed, 1995: 237-238.

2. Boyes RJ, Kruse JA. Nasogastric and nasoenteric intubation. Crit Care Min 2002; 8: 865-878

3. Miller KS, Tomlinson JR, Sahn SA. Pleuropulmonary complications of enteral tube feedings. Two reports, review of the literature, and recommendations. Chest 1985; 88: 230-233.

4. Thomas D, Cummin D, Falcone RE. Accidental pneumothorax from a nasogastric tube. N Engl J Med 1996; 335: 1325-1326.

5. Hendry PJ, Akyurekll, McIntire R, Quarrington A, Keo W. Bronchopleural complications of nasogastric feeding tubes. Crit Care Med 1986; 14 (10): 892-894

6. Kindop AS, Drover JW, Heyland DK. Capnograhy confirms correct feeding tube placement in intensive care unit patients. Can J Anesth 2001; 48: $705-10$

\section{Trombosis venosa profunda de miembro superior derecho tras fractura de clavícula}

\section{Sr. Director:}

La trombosis venosa profunda se describe frecuentemente asociada a alteraciones en el sistema venoso profundo de miembros inferiores. Sólo del 1-4\% de los casos, se origina en el territorio de la vena cava superior afectando principalmente a las venas axilar ó subclavia $(1,2)$. Se encuentra asociada a catéteres venosos centrales, estados de hipercoagulabilidad, neoplasias, anticonceptivos orales y compresiones extrínsecas. Dentro de este último grupo se encuentran las debidas a costillas cervicales, el síndrome de compresión de los escalenos, y las fracturas de clavícula con callo de formación (1-4). Presentamos un caso de trombosis venosa profunda en miembro superior derecho secundaria a fractura previa de clavícula.

Caso: Varón de 29 años de edad sin antecedentes personales de interés. El mes previo al ingreso había consultado tras sufrir fractura de clavícula derecha en un accidente de tráfico. Fue dado de alta con analgesia e inmovilización mediante vendaje de Velpeau. No realizaba ningún tratamiento. Consultó por presentar en la última semana, dolor e inflamación en miembro superior derecho (MSD). No refería disnea, hemoptisis ni dolor torácico. No síndrome constitucional.

En la exploración destacaban inflamación y dolor a la palpación en miembro superior derecho, sin aumento de temperatura. Los pulsos periféricos estaban conservados. No se palpaban adenopatías periféricas. El resto de la exploración fue normal.

El hemograma, la bioquímica y el estudio de coagulación fueron normales. Se realizó estudio de trombofilia en el que los valores de proteínas $\mathrm{C}$ y $\mathrm{S}$, antitrombina III, anticardiolipina, y homocisteina fueron normales, y las determinaciones de anticoagulante lúpico, mutación del gen de la protrombina, factor $\mathrm{V}$ de Leyden negativos. En la radiografía de tórax se apreciaba fractura en resolución de la clavícula derecha sin otros hallazgos significativos. El eco-doppler venoso de MSD informó de trombosis venosa profunda a nivel de vena axilar distal y vena humeral. Se descartó la existencia de tromboembolismo pulmonar.

El paciente evolucionó favorablemente tras realizar tratamiento anticoagulante con enoxaparina y acenocumarol. En revisiones posteriores ha permanecido asintomático.

La trombosis venosa profunda presenta una incidencia anual de aproximadamente $0,1 \%$ (5). La originada en las extremidades superiores ha sufrido un incremento en su presentación de forma secundaria al aumento en la utilización de catéteres venosos centrales para la administración de quimioterapia, diálisis y nutrición parenteral. En los pacientes más jóvenes, se ha objetivado que el $56 \%$ de los casos de enfermedad tromboembólica se relacionan con etiología congénita o con la presencia de anticuerpos antifosfolípidicos (6).

Sin embargo en nuestro caso, se descartó la existencia de neoplasia y el estudio de trombofilia fue normal. Por este motivo, debemos suponer que la TVP de MID se desarrolló de forma secundaria a la compresión de la zona debida al vendaje y a la formación del callo de fractura.

La sintomatología es inespecífica. Las manifestaciones clínicas más habituales son dolor, hinchazón y edema, aunque en la mayoría de los casos los pacientes se encuentran asintomáticos, por lo que se retrasa el diagnóstico y el tratamiento.

El tromboembolismo pulmonar es la complicación más frecuente. Se recoge en el 9\% (7) o 20\% (2) de los casos, según la literatura revisada. En otros casos, podemos encontrar síndrome postflebítico, tromboflebitis, e incluso en los más graves, gangrena en la extremidad superior afecta (1). En nuestro caso, no se presentó ninguna complicación posterior.

Para realizar el diagnóstico definitivo son imprescindibles las pruebas de imagen. La flebografia radiológica sigue siendo la técnica gold standard en esta patología, pero debido a su elevado coste y agresividad se realiza en pocas ocasiones. Mediante la realización del eco-doppler venoso se obtiene una elevada sensibilidad y especificidad, por lo que se considera de elección en la mayoría de los casos (4).

El tratamiento de elección es la heparina de bajo peso molecular seguida de anticoagulación oral. Se debe administrar durante al menos tres meses, manteniendo controles periódicos 
de INR entre 2-3. La duración de la anticoagulación depende de la etiología de la TVP, se mantiene de forma indefinida en los casos en los que persiste la causa que la ocasionó. En algunos casos, se propone el tratamiento trombolítico, ya que en infusión directa a través del catéter resultaría muy eficaz. Sin embargo el riesgo de sangrado es mayor que con heparina de bajo peso molecular y no se ha demostrado que reduzcan el síndrome postflebítico, por este motivo se consideran de segunda opción terapéutica $(1-3,5)$.

\section{D. Mañas García, C. Roldán Machado, J. Gijón Rodrí-} guez

Servicio de Medicina Interna. Complejo Hospitalario. Ciudad Real
1. Kommareddy A, Zaroukian MH, Hassouna IH. Upper extremity deep venous thrombosis. Semin Thromb Hemost 2002; 28: 89-99.

2. Sabeti S, Schillinger M, Mlekusch W, Haumer M, Ahmadi R, Minar E. Treatment of subclavian-axillary vein thrombosis: long-term outcome of anticoagulation versus systemic thrombolysis. Thromb Res 2002; 108: 279-285.

3. Joffe HV, Goldhaber SZ. Upper-extremity deep vein thrombosis. Circulation 2002; 106: 1874-1880.

4. Prandoni P, Polistena P, Bernardi E, Cogo A, Casra D, Verlato F,et al Upper-extremity deep vein thrombosis. Risk factors, diagnosis and complications. Arch intern Med 1997; 157: 57-62.

5. Bates SM, Ginsberg JS. Treatment of deep vein thrombosis. N Engl J Med 2004; 351: 268-277

6. García-Fuster MJ, Fernández C, Forner MJ, Vaya A. Estudio prospectivo de los factores de riesgo y las características clínicas de la enfermedad tromboembólica en pacientes jóvenes. Med Clin (Barc) 2004; 123: 217 219.

7. Mustaf S, Stein PD, Patel KC, Otten TR, Holmes R, Silbergleit A. Upper extremity deep venous thrombosis. Chest 2003; 123: 1953-1956. 